\title{
Lightcurve studies and magnetic activities of several eclipsing binaries
}

\author{
Xianming L. Han ${ }^{1,2}$, Liyun Zhang ${ }^{1}$, Qingfeng $\mathbf{P i}^{1}$ and Daimei Wang ${ }^{1}$ \\ ${ }^{1}$ Department of Physics, College of Science, Guizhou University and NAOC-GZU-Sponsored \\ Center for Astronomy, Guizhou University, Guiyang 550025, P.R. China \\ ${ }^{2}$ Dept. of Physics and Astronomy, Butler University, Indianapolis, IN 46208, USA \\ email: xhan@butler.edu
}

\begin{abstract}
Late-type stars with thick convective zones and rapid rotations exhibit magnetic activity phenomena, such as starspots, plages, and flares. However, in many such kinds of eclipsing binaries, the details of the active phenomena are not well understood. In order to improve our understanding of stellar magnetic activities, we are carrying out an extensive study of the magnetic activities of eclipsing binaries by multi-color CCD photometry using several telescopes (SARA $90 \mathrm{~cm}$ at KPNO, and NAOC $85 \mathrm{~cm}$ and $60 \mathrm{~cm}$ telescopes at Xinglong). In this paper, we will present our preliminary results with revised orbital parameters, starspot parameters, and stellar flare events on DV Psc and BX Tri.
\end{abstract}

Keywords. stars: individual (DV Psc, BX Tri), stars: flare, stars: activity, binaries: eclipsing.

\section{Introduction}

Eclipsing binaries with Late-type stars exhibit magnetic activity phenomena (starspot, plage, and flare) because of their thick convective zones and rapid rotations. Studying the details of the active phenomena will provide us stellar magnetic activity properties and evolution. BX Tri (GSC 2314:530, NSVS 6550671) was discovered to be an eclipsing binary system by the Northern Sky Variability Survey (Wozniak et al. 2004), and attracted our attention because it is the shortest period dM system and flare events (Dimitrov \& Kjurkchieva, 2010; Zhang et al.2014). DV Psc is a detached, near-contact binary system (Zhang \& Zhang 2007). The first flare-like event of DV Psc was detected by Zhang et al. (2010). DV Psc attracted our attention (Pi et al. 2014, etc) because it exhibits frequent flare events and changing orbital period. In this paper, we present our preliminary results of BX Tri and DV Psc.

\section{Observation}

We observed BX Tri in December of 2012 using the $85 \mathrm{~cm}$ telescope located at the Xinglong Station, National Astronomical Observatory of China, and again in November 11, 15, 19 of 2014 using the 90cm SARA (Southeastern Association for Research in Astronomy) telescope located at Kitt Peak National Observatory (KPNO) in Arizona, USA. The results from the December 2012 observations are shown in Fig 1.

We observed DV Psc system in 2013 and 2014, using a 1-m telescope at Yunnan Observatory in Kumin, China, the 90-cm SARA telescope at Kitt Peak National Observatory in USA, and the 60-cm telescope at Xinglong Station of the National Observatory of China. The resulting lightcurves are also shown in Fig 1. 

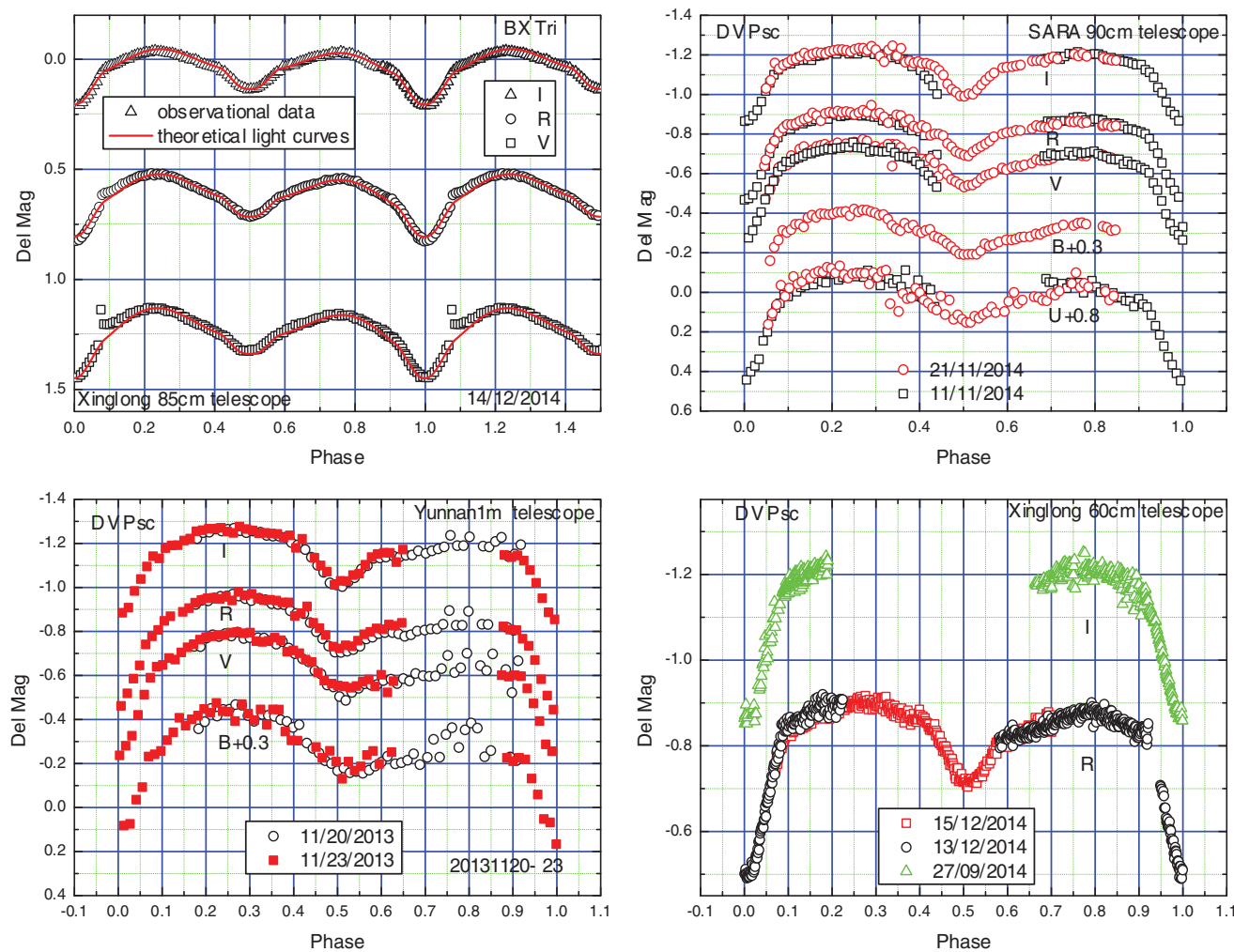

Figure 1. VRI light curves of BX Tri from our 2012 observations (Left) and DV Psc in 2012-2014 (right).
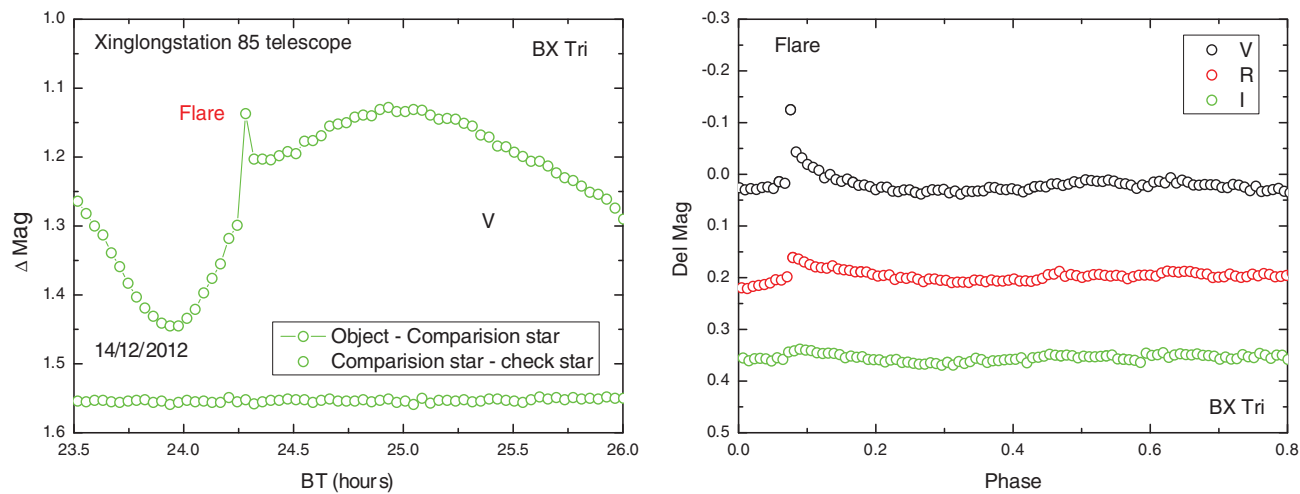

Figure 2. The flare of BX Tri in V, R and I bands. The left figure is the observational data in $\mathrm{V}$ band. The right figure represents the difference between the observational data and their corresponding theoretical values.

\section{Analyses}

We analyzed our light curves of BX Tri using the 2003 version of the Wilson-Devinney program (Wilson \& Devinney 1971; Wilson \& Van Hamme 2004). We note that the secondary maximum in the light curve is highly distorted. We obtained the starspot parameters using WD Program (See Figures. 1 \& 3.). This distortion can be explained by a star spot on the equator of the primary star, at a longitude of 281 degrees, with a 

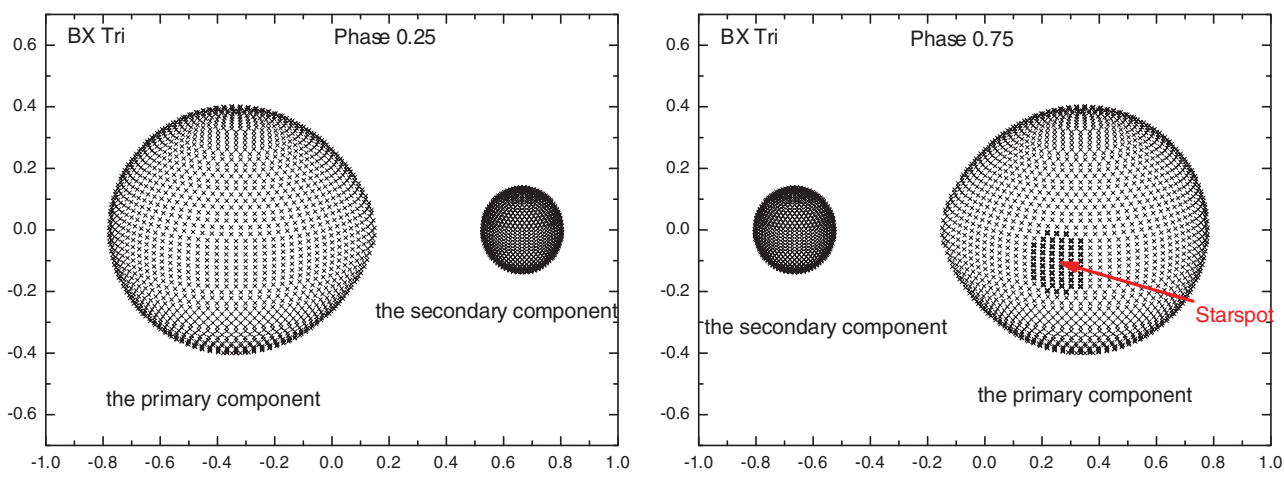

Figure 3. Starspot on the primary of BX Tri in phases 0.25 and 0.75 .

radius of 15.7 degrees, and a temperature of $3550 \mathrm{~K}$ ( 0.95055 of the normal temperature). This starspot is similar in size, location and temperature to that reported previously by Zhang et al.(2014), indicating that the starspot is semipermanent. We detected a large flare in our 2012 observations. Fig. 2 (left) shows the observed light curve in V-band. To better show the effect of the flare, we subtracted the fitted values from the observed data, and plotted the results in Fig. 2 (right). The flare is most prominent in the V-band as expected, with a magnitude of 0.15 mag. This is the largest flare ever detected for BX Tri. We did not detect any flare events in our 2014 observations (Zhang et al. 2014).

We did not detect any flares of DV Psc in any of these observation sessions, indicating that this binary system may be in a period of minimal activities. We did not analyze the light curve of DV Psc using WD program because of no flare event.

\section{Conclusions}

We observed BX Tri in 2013 and 2014, and detected the largest flare with a magnitude of $0.15 \mathrm{mag}$ in $\mathrm{V}$ band. We used the starspot model to successfully explain the asymmetry in its light curve. We also observed DV Psc in 2014, and obtained its U-band light curve for the first time in addition to its BVRI light curves. Its lightcurves show strong asymmetry between the primary and secondary maxima.

\section{Acknowledgements}

We are very grateful to the observing assistants at the three telescopes during our observations. This work was supported by the Joint Fund of Astronomy of the National Natural Science Foundation of China and the Chinese Academy of Sciences Nos. U1431114 and 11263001, and science and technology innovation team of Guizhou province (2015)4017.

\section{References}

Dimitrov, D. P. \& Kjurkchieva, D. P. 2010, MNRAS, 406, 2559

Pi, Q. F., Zhang, L. Y., Li, Z. M., et al. 2014, AJ, 147, 50

Wilson, R. E. \& Devinney E. J. 1971, ApJ, 166, 605

Wilson, R. E. \& Van Hamme, W., 2004, Computing Binary Star Observables

Wozniak, P. R., Vestrand, W. T., Akerlof, C. W., et al. 2004, AJ, 127, 2436

Zhang, L. Y. \& Gu, S. H. 2007, A\&SA, 471, 219

Zhang, L. Y., Pi, Q. F., \& and Yang, Y. G. 2014, MNRAS, 442, 2620

Zhang, L. Y., Zhang, X. L., \& Zhu, Z. Z. 2010, New Astron., 16, 362

Zhang, X. B. \& Zhang, R. X. 2007, MNRAS, 382, 1133Z 